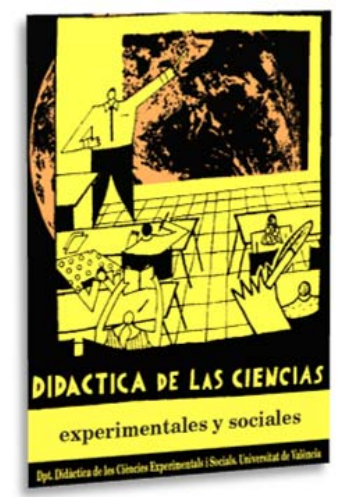

\title{
Educación no formal. El papel del profesorado en las visitas didácticas a los museos
}

\author{
Non-formal education. The role of teachers in \\ the didactic visits to museums
}

DOI: $10.7203 / D C E S .37 .13307$

\author{
José-Manuel González González \\ Universidad de Zaragoza, joseman@unizar.es \\ ORCID iD: http://orcid.org/0000-0002-0383-2721 \\ María-Teresa Becerra Traver \\ Universidad de Extremadura, mbectra@unex.es \\ ORCID iD: http://orcid.org/0000-0003-2771-3417
}

\begin{abstract}
RESUMEN: Los museos, desde hace décadas, imbuidos de las ventajas de la didáctica, reciben cada vez a más escolares. Sin embargo, hay escasos estudios que nos hablen de las acciones que llevan a cabo los profesores cuando, con sus alumnos, visitan un museo. En este trabajo queremos conocer en qué medida el profesorado o los maestros y maestras se involucran en todo lo que rodea la actividad de visitar un museo. A través de la cumplimentación de una encuesta en Extremadura se ha hallado que el profesorado realiza un trabajo previo y posterior a la visita, aunque pueda haber cierta parcialidad en sus respuestas.
\end{abstract}

PALABRAs Clave: educación, didáctica, museo, profesorado, actividades.

ABSTRACT: For decades, museums, permeated by the advantages of didactics, have been receiving an increasing number of schoolchildren. However, there are only a few studies that present the actions carried out by teachers who visit museums with their students. In this work, we want to determine to what extent teachers are involved in everything that concerns the activity of visiting a museum. Through the completion of a survey in Extremadura, we have found out that teachers carry out work before and after the visit, although there may be some bias or partiality in their answers.

KEYWORDS: education, didactics, museum, teachers, activities.

Fecha de recepción: noviembre de 2018

Fecha de aceptación: junio de 2019

Los autores agradecen su colaboración a los museos y profesores participantes. 


\section{INTRODUCCIÓN}

La educación no formal está cobrando un especial interés en los últimos cincuenta años, si bien con la irrupción de las nuevas tecnologías cada vez es más evidente su trascendencia en el aprendizaje de la sociedad actual. No hace mucho, se señalaba en un magnífico artículo compilatorio, que la diferencia educativa entre la educación formal y la informal sólo tenía un carácter jurídico y que debía haber una conexión y una relación cada vez más estrecha entre ambas, para intercambiar experiencias (Colom, 2005). Es por lo tanto vital que empecemos a tomar muy en serio la formación que se recibe fuera del aula, en este caso en centros culturales especializados.

Los museos, que fueron uno de los pioneros en la educación no reglada, han crecido exponencialmente en las últimas décadas, indicando una mayor concienciación cultural y especificando el interés institucional por custodiar colecciones que difundan el conocimiento de diversos aspectos científicos. La mayoría cuentan ya con departamentos didácticos o personal especializado al respecto. A pesar de ello:

En nuestro entorno, es difícil encontrar un museo que tenga una oferta moderna; la mayoría de los programas giran en torno a visitas guiadas, talleres y fichas didácticas. Todos estos tipos de formatos se vienen utilizando en museos desde hace al menos 50 años. Un hecho más. No conocemos ningún museo por estos lares que mantenga una política continuada interna o externa de evaluación o control de calidad de la oferta de estos programas infantiles. (Pol y Asensio, 2006).

Se habla hoy en día de que los museos no sólo proporcionan interesantes actividades para los alumnos, sino también para profesores, con cuadernos didácticos exclusivos para los maestros, cursos de formación, talleres para adultos, etc. En el caso de los museos de ciencias ya se ha estudiado su interés por parte del profesorado (Morentin, 2015; Porta y Mato, 2016), pero también en relación con las ciencias sociales y el patrimonio ha habido un estudio de caso que señala la necesidad de fomentar el conocimiento patrimonial del entorno más cercano (Molina y Muñoz, 2016). Además, varios estudios señalan que cuando las experiencias de aprendizaje externas a la escuela se incluyen en el currículum, se incrementa el impacto educativo de las visitas (Eshach, 2006). Dicho de otra manera, las exposiciones pierden su eficacia educativa si no se parte de un vínculo con el currículum escolar (Sánchez, 2013). Sin embargo, no siempre se practican o se hace uso de ellos, y a veces se opta por el camino más fácil:

En contra de lo que todo el mundo piensa, los profesores no eligen las actividades en función de los contenidos o de su adecuación a los curricula, sino (pásmense) de las facilidades de servicios que les ofrece la institución, como la existencia de guías que se hagan cargo de los escolares, aparcamientos para los autobuses, cafeterías y comedores, horarios, cercanía al centro o a las conexiones, etc. (Pol y Asensio, 2006: 14).

Muchos profesores que hacen actividades extraescolares con sus alumnos son incapaces de decir el propósito que les guía en su actividad fuera del aula, y bastantes no lo consideran relacionado con el curriculum, aunque sí enriquecedor; así mismo, según se ha investigado, casi todos hacen salidas o excursiones sin preparación previa o retroalimentación posterior (Kisiel, 2003).

Los estudios de Kisiel, centrados en el uso de rúbricas, han incidido en que tanto una visita previa como actividades de seguimiento posteriores ayudan, y mucho, en el aprendizaje y el recuerdo de contenidos o procedimientos mostrados por los museos. En el caso de México, y según la encuesta realizada entre 2008 y 2009 por el Sistema de Información Cultural del Gobierno, la razón principal de la visita a los mismos fue: en un 32\% porque se lo pidieron en la escuela, y en un 7\% para educar a los niños (véase: https://sic.cultura.gob.mx/documentos/1250.pdf [17 de octubre de 2018]). 
Por otro lado, el uso de los cuestionarios o las encuestas está generalizado en los estudios de ciencias sociales. Como es conocido, permiten analizar aspectos cuantitativos o cualitativos, extrayendo importantes conclusiones. En los museos también ha sido utilizada esta herramienta, como muestra de la preocupación desde la propia institución o desde la administración, sobre todo para examinar al público que asiste (Verde, 1993) o para mejorar el interés que despiertan y conocer las motivaciones que impulsan a los visitantes y su grado de satisfacción.

Aunque algunos países puedan estar más avanzados en esta cuestión, lo cierto es que se denotan carencias en toda Europa, como sugiere un importante estudio realizado por la Dirección General de Patrimonio Cultural de Dinamarca, donde se afirmaba que una gran mayoría de los 130 museos analizados no efectuaban una evaluación sistemática de sus actividades educativas, y que más de un $30 \%$ ni siquiera evaluaba su enseñanza, si bien el 45\% interrogaba sobre la satisfacción del docente (VV. AA., 2008).

La preocupación en España ha fomentado varios encuentros profesionales, algunos organizados por el Ministerio de Cultura, como las Jornadas DEAC, de Departamentos de Educación y Acción Cultural de museos, con casi veinte reuniones desde 1980. Puede verse su origen y un resumen histórico en https:/www.macba.cat/uploads/publicacions/desacuerdos/textos/ desacuerdos_6/Aida_Sanchez.pdf [30 de septiembre de 2018]; e información muy completa sobre la última y otras en https://www.museodelprado.es/actualidad/actividades [3 de octubre de 2018]. También son interesantes los congresos últimamente llevados a cabo por el Museo Thyssen de Madrid, con el título Los Museos en la educación (https://www.educathyssen.org/centroestudios/educacion-museos [3 de octubre de 2018]). También se han dado proyectos de investigación, alguno de los cuales se han convertido en observatorio científico (Fontal, 2016). En los museos de Extremadura existen departamentos didácticos desde la llegada de la democracia y se han publicado relatos y reflexiones sobre su labor (Caldera de Castro, 1998; Caldera de Castro y Nodar, 2005).

Ahora bien ¿Quién debe realizar las encuestas? Aunque las opiniones son diversas, y hay que atender a si tienen un enfoque generalista o crítico-transformador, es frecuente que la propia administración de Cultura o el museo elabore un seguimiento estadístico, aunque en ocasiones parece conveniente que la evaluación sea ejecutada por 'personas ajenas al propio museo' (Verde, 1993; Calaf, San Fabián y Gutiérrez, 2017) .

En ese sentido, justificamos nuestra aportación y abordamos el estudio del papel del profesor en las visitas didácticas a los museos de Extremadura. Ante esta premisa, queremos conocer si los propios profesores trabajan con los alumnos antes y después de visitar el museo, lo que nos reportará conclusiones sobre cómo abordan este tipo de actividades especiales fuera del aula, dentro de la educación no reglada. En concreto, queremos obtener respuesta a preguntas como: ¿Se toma en serio el profesorado la visita a un museo? ¿Prepara una unidad didáctica al respecto? ¿Les resultan útiles a los profesores los museos extremeños?

\section{MÉTODO}

\subsection{Muestra}

Teniendo en cuenta los museos implantados en la región, pertenecientes a la Red de Museos de Extremadura, (http://www.arqueotur.org/redes/red-de-museos-de-extremadura.html [5 de septiembre de 2018]), se optó por hacer un listado y enviar una petición a aquellos museos más visitados según el Instituto de Estadística de Extremadura (https://ciudadano.gobex.es/web/ieex/ banco-de-datos?vistaFactorId=2769062 [1 de noviembre de 2018]), siendo doce los seleccionados, aquellos con más de diez mil visitantes. No obstante, finalmente, sólo siete contestaron afirmativamente a nuestra petición:

- Museo de Cáceres. 
- Fundación Helga de Alvear, Cáceres.

- Museo Vostell Malpartida de Cáceres.

- Museo Extremeño e Iberoamericano de Arte Contemporáneo (MEIAC), Badajoz.

- Museo Arqueológico Provincial de Badajoz.

- Museo Etnográfico González Santana de Olivenza.

- Museo Santa Clara de Zafra.

La tipología predominante es exclusivamente la de un museo cultural, especializado en historia y arte, dentro del campo de las ciencias sociales y humanas. Uno de los museos analizados tiene varias secciones diferentes dentro de su contenido. Así, hay 3 museos de arte contemporáneo, 1 museo arqueológico, 1 museo etnográfico, 1 museo religioso y 1 que tiene arqueología, etnografía y bellas artes. La mayoría tienen un carácter fundamentalmente regional. De los museos participantes, en el momento del estudio, 4 sólo mostraban al público una colección permanente, 1 combinaba una colección permanente con exposiciones temporales, y 2 exhibían únicamente exposiciones temporales.

Los siete museos seleccionados recibieron 75 grupos escolares, cuyos profesores decidieron colaborar en la encuesta que hicimos al efecto. El museo que más grupos escolares recibió durante los tres meses que se distribuyó la encuesta (entre abril y junio de 2016) fue el MEIAC (44\% de profesores con su grupo de alumnos). Esto, probablemente fue debido a su cercanía a muchos centros educativos, pero también por los talleres que disponen.

TABLA 1. Profesores participantes en el estudio.

\begin{tabular}{ccccccccc}
\hline \multirow{2}{*}{ Museos } & MEIAC & $\begin{array}{c}\text { González } \\
\text { Santana }\end{array}$ & $\begin{array}{c}\text { Museo de } \\
\text { Cáceres }\end{array}$ & $\begin{array}{c}\text { Arqueológico } \\
\text { de Badajoz }\end{array}$ & $\begin{array}{c}\text { Helga } \\
\text { de } \\
\text { Alvear }\end{array}$ & Vostell & $\begin{array}{c}\text { Santa } \\
\text { Clara }\end{array}$ \\
\hline $\begin{array}{c}\text { Profesores } \\
\text { visitantes }\end{array}$ & $\mathrm{N}$ & 33 & 13 & 12 & 6 & 6 & 4 & 1 \\
\cline { 2 - 9 } & 44 & 17 & 16 & 8 & 8 & 5 & 2 \\
\hline
\end{tabular}

A estos museos asistieron un total de 75 profesores que impartían clase en Extremadura, lo que, además de indicar que el interés de los mismos es fundamentalmente regional, da unidad a la muestra. Los profesores participantes en el estudio, y que visitaron los museos mencionados, eran de la especialidad de Infantil (6.7\%) Primaria (61.3\%), Secundaria (25.3\%), Educación para Adultos (2.7\%) y Bachillerato (4\%). El 71.2\% eran mujeres y el 28,8\% hombres, siendo la media de edad global de 45,7 años. De los profesores de los centros escolares, 58 trabajaban para centros públicos y 17 eran privados o concertados (77.3\% y 22.7\%, respectivamente).

\subsection{Diseño}

El estudio se fundamentó en un estudio descriptivo de encuesta, basado en las respuestas obtenidas en un cuestionario previamente elaborado con objeto de valorar la visita didáctica desde el punto de vista de los profesores, y su opinión al respecto. Se partió de un modelo desde cero, con preguntas variadas de tipo cuantitativo y cualitativo El proceso de validación se llevó a cabo mediante grupos de discusión con expertos internos y externos, especialistas en educación y museos. Las medidas de validez adoptadas tuvieron en cuenta las opiniones de los encuestadores y los encuestados, con una primera entrega de prueba entre el personal del área educativa de los museos. 


\subsection{Materiales}

Para llevar a cabo este estudio, como se ha comentado previamente, se diseñó un cuestionario que fue sometido a la valoración de expertos (profesores, alumnos de primaria y secundaria y técnicos de museos), mediante grupos de discusión. Se trata de un cuestionario autocumplimentado formado por 20 ítems agrupados en torno a tres secciones: 1) Datos de Identificación; 2) Visita al museo y; 3) Información sobre la visita a este museo.

La primera sección pretende recoger datos generales de identificación y clasificación, como edad, sexo, nivel educativo... Con respecto a la segunda sección, se extrae información relativa, sobre todo, a las actividades que realizan en el aula los profesores con sus alumnos antes y después de visitar el museo. La mayoría de las preguntas de esta sección son cerradas dicotómicas (sí o no). La tercera sección cuenta con opciones de respuesta de una escala tipo Likert (1932) con cinco niveles de acuerdo o desacuerdo, siendo 1=Totalmente en desacuerdo, 2=En desacuerdo, 3=Ni de acuerdo ni en desacuerdo, 4=De acuerdo y 5=Totalmente de acuerdo. En esta sección se valoran aspectos como la organización de la visita, los recursos y contenidos trabajados durante la visita y el aspecto o aprecio global de la visita al museo. Además, en esta tercera sección se plantearon dos preguntas abiertas sobre las actividades que más le han gustado o le gustaría que sus alumnos trabajasen en el desarrollo de la visita. En el anexo 1 puede verse el cuestionario utilizado.

\subsection{Procedimiento}

La cumplimentación de los cuestionarios se llevó a cabo entre los meses de abril y junio de 2016. Dicho cuestionario se envió a doce museos y centros de interpretación de los cuales siete fueron los que finalmente pudieron participar. No obstante, consideramos que se trata de una muestra bastante equilibrada por su distribución geográfica y tipología; hay que tener en cuenta la dificultad que suponía rellenar una encuesta por escrito al final de la visita o actividad, dado el tiempo disponible por los docentes antes de volver al aula. La duración estimada para rellenarla iba de 3 a 5 minutos. De los siete museos participantes, el número de profesores que accedieron a colaborar en la cumplimentación de la encuesta se pueden consultar en la tabla 1.

Una vez recogidos los cuestionarios cumplimentados, por los profesores participantes que visitaron los museos mencionados, se procedió a su análisis por parte de los autores de este estudio. Para ello, se realizó en el editor de datos de SPSS una base que arrojó más de 2.000 reseñas en global relacionadas con los ítems de la encuesta. Esto nos llevó a observar la frecuencia con que aparecía cada respuesta en cada pregunta y, por lo tanto, a codificar la información y a agrupar los datos cuantitativos en frecuencias y porcentajes. No sólo se llevó a cabo el análisis teniendo en cuenta el conjunto de participantes sino que hicieron cruces de análisis entre las diferentes preguntas para ver su relación.

Finalmente, se realizaron tablas para sintetizar la información, así como representaciones gráficas de los datos. A partir de aquí se pudo realizar una interpretación descriptiva de la información dentro del contexto en el que fue recogida y por tanto, extraer unas conclusiones.

\section{RESUltados}

El procedimiento anteriormente descrito dio lugar a una amplia base de datos cuantitativos y cualitativos, cuyos resultados se muestran y explican detalladamente en dos bloques: 1) por un lado, los resultados extraídos de cada una de las secciones que componen el cuestionario y por otro lado, 2) los análisis resultantes del cruce de distintas variables. 


\subsection{Secciones del cuestionario}

En este apartado se muestran los resultados extraídos de las tres secciones mencionadas anteriormente del cuestionario.

\section{Sección 1. Datos de identificación}

Los datos proporcionados y los aquí mostrados guardan el anonimato de los encuestados. Las combinaciones que utilizamos nos permiten conocer las características de los participantes sin desvelar su identidad.

De los 75 profesores, 17 venían de la provincia de Cáceres y 58 de la de Badajoz (22.7 frente al 77.3\%). El centro escolar con más profesores visitantes fue un centro público de primaria de Badajoz, con 13 profesores, lo que pudo influir también en el porcentaje antedicho.

De los docentes, 52 son mujeres y 21 hombres (71.2 frente al $28.8 \%$ ), 2 no contestan a qué sexo pertenecen. La media de edad es de 45.7 años. En cuanto a grupos de edad, hemos establecido cuatro rangos para clasificar por ciclos o generaciones: entre 25 y 39 años (18 profesores), entre 40 y 54 años (27 profesores), entre 55 y 65 años (19 profesores) y los que no dijeron la edad (11 profesores). 25 y 65 años son los dos extremos de edad entre los encuestados.

\section{Sección 2. Visita al museo}

Con respecto al ítem Es mi primera visita a este museo, el 88\% afirma que no es su primera visita y el $12 \%$ que sí es su primera visita. Sin embargo, el 30.7\% indica que sí es la primera visita que hace al museo con el colegio o instituto. Un 69.3\% sí había programado visitas al museo con sus estudiantes.

Después de la visita al museo los profesores participantes consideran que sí volverán a repetir la visita el curso que viene (el 93\%).

El 68.5\% de profesores afirman haber trabajado previamente con los alumnos algo en clase antes de ir al museo. En concreto, señalan actividades como haber hecho una visita virtual, información sobre el tema que iban a ver (Cubismo, Prehistoria, Arte Abstracto...), utilización de vídeos e imágenes, fichas didácticas sobre la organización del museo y las distintas piezas y objetos que pueden los alumnos encontrarse, entre otras. Así mismo, también el 74.6\% piensa evaluar en clase lo que han aprendido sus alumnos en el museo. Indican, para ello, actividades como hacerles preguntas oralmente de lo que han aprendido, hacer un debate, completar unas fichas, pedirles una composición escrita de lo que han visto, hacer un dibujo...

Cuando se les preguntó en el cuestionario que rodeasen con un círculo qué habían hecho sus alumnos en el museo, el 49.3\% marcó más de una opción entre las establecidas (visita guiada, taller, audiovisual, hoja de ejercicios...), seguido del 48\% que indicó que habían realizado una visita guiada.

\section{Sección 3. Información sobre la visita a este museo.}

Como se puede apreciar en la tabla 2 y gráfico 1, con respecto a las ítems con respuestas con cinco niveles de acuerdo o desacuerdo, la mayoría del profesorado que asistió a estos museos está totalmente de acuerdo con la información recibida del museo, tanto a la organización y duración de la visita como al material proporcionado y a las actividades didácticas planteadas. Tanto es así, que el 82.4\% de profesores recomiendan la visita a otros compañeros, familiares o amigos. 
TABLA 2. Sección 3 del cuestionario. Información sobre la visita a este museo.

(1=Totalmente en desacuerdo, $2=$ En desacuerdo, $3=\mathrm{Ni}$ de acuerdo ni en desacuerdo, 4=De acuerdo y 5=Totalmente de acuerdo)

\begin{tabular}{|c|c|c|c|c|c|}
\hline & 1 & 2 & 3 & 4 & 5 \\
\hline \multicolumn{6}{|l|}{ Guía } \\
\hline $\begin{array}{l}\text { La información recibida por el guía ha sido correcta y } \\
\text { adaptada a mis alumnos. }\end{array}$ & $1.3 \%$ & $1.3 \%$ & $4 \%$ & $25.3 \%$ & $68 \%$ \\
\hline La organización de la visita me ha gustado. & $1.4 \%$ & $1.4 \%$ & $1.4 \%$ & $23.3 \%$ & $72.6 \%$ \\
\hline \multicolumn{6}{|l|}{ Recursos y contenido } \\
\hline La duración de la visita es la adecuada. & $1.4 \%$ & $0 \%$ & $1.4 \%$ & $21.6 \%$ & $75.7 \%$ \\
\hline Los textos y objetos se adaptan a mis alumnos. & $1.4 \%$ & $2.7 \%$ & $12.2 \%$ & $24.7 \%$ & $58.1 \%$ \\
\hline Se han hecho actividades didácticas. & $15 . \%$ & $4.5 \%$ & $10.6 \%$ & $15.2 \%$ & $54.5 \%$ \\
\hline \multicolumn{6}{|l|}{ Valoración global } \\
\hline $\begin{array}{l}\text { Como profesor/a. me ha gustado lo que se ha propuesto } \\
\text { por el museo. }\end{array}$ & $1.4 \%$ & $1.4 \%$ & $4.2 \%$ & $23.6 \%$ & $69.4 \%$ \\
\hline $\begin{array}{l}\text { He aprendido cuestiones que desconocía } \\
\text { enriqueciéndome como profesor. }\end{array}$ & $1.4 \%$ & $5.4 \%$ & $6.8 \%$ & $23 \%$ & $63.5 \%$ \\
\hline Recomendaré la visita a compañeros/familiares/amigos. & $1.4 \%$ & $0 \%$ & $1.4 \%$ & $14.9 \%$ & $82.4 \%$ \\
\hline
\end{tabular}

GRÁFICO 1. Sección 3 del cuestionario. Información sobre la visita a este museo.

(1=Totalmente en desacuerdo, 2=En desacuerdo, 3=Ni de acuerdo ni en desacuerdo, 4=De acuerdo y 5=Totalmente de acuerdo)

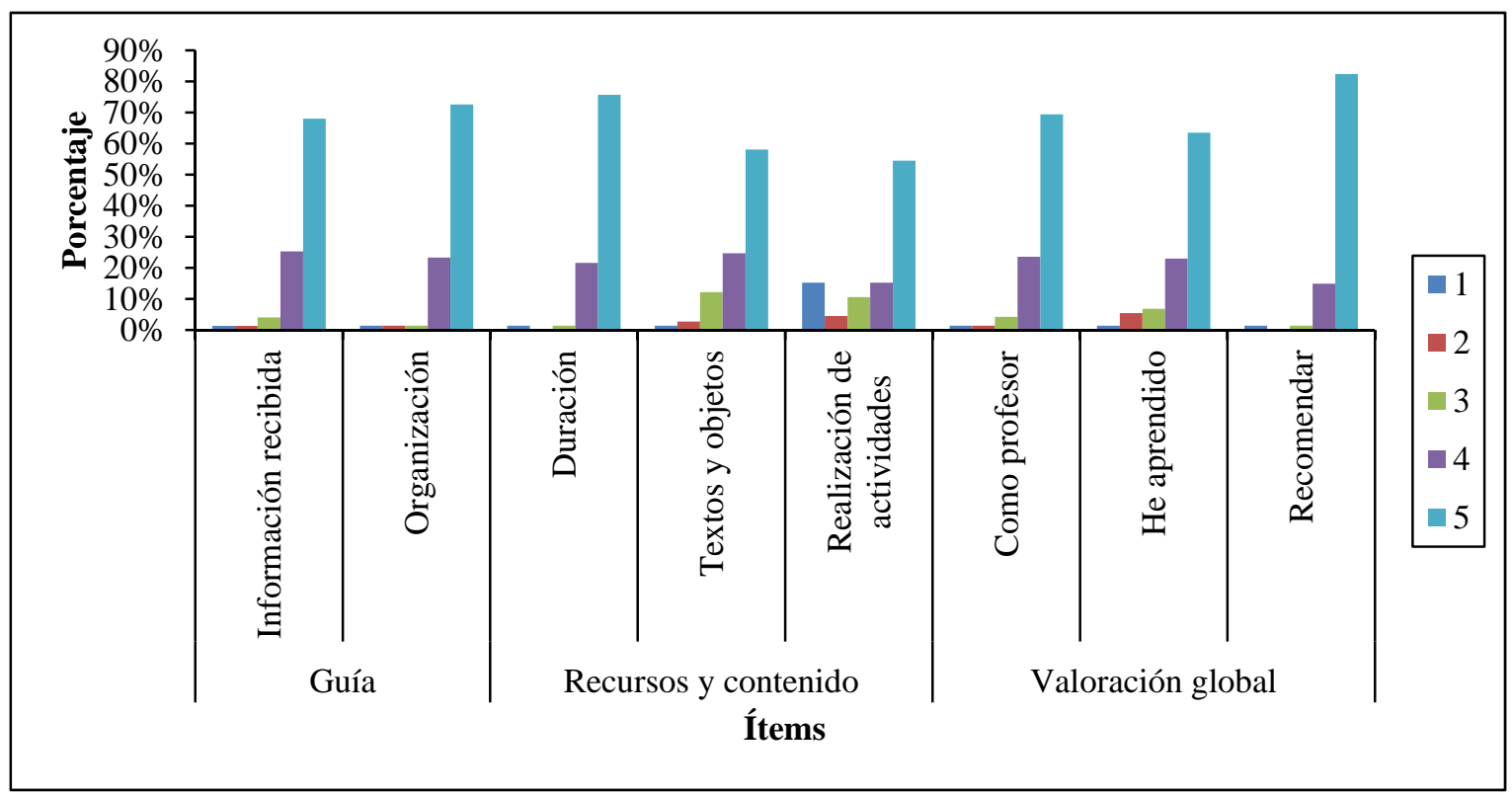

En cuanto a las preguntas abiertas de esta sección, por un lado, en la pregunta La actividad que más me ha gustado ha sido, algunos profesores resaltan en sus respuestas la profesionalidad del guía o el atractivo de algunas salas concretas (caso del Aljibe de Cáceres); en otros casos, que se les pidiera a los alumnos redactar lo vivido. En algunos museos como el MEIAC se resalta su taller y la dramatización o juego de roles que se hace, en el Arqueológico de Badajoz el cuaderno u hoja de actividades; en otros museos afirman que les ha gustado todo. Por otro lado, en la pregunta $L a$ actividad que me gustaría que mis alumnos hicieran cuando visitaran un museo es, muchos opinaban que la participación del alumnado, exponiendo sus opiniones o realizando talleres didácticos. Otros pedían más interactividad o elaborar una ficha de trabajo durante la visita, visualizar algún audiovisual, búsqueda del tesoro o realizar cerámica; también pedían realizar collages, juego de roles o talleres manipulativos. 


\subsection{Cruce de variables}

Además de los resultados anteriormente analizados, queríamos ver también la relación que había entre las siguientes variables: 1) trabajo previo en el aula y evaluación según el nivel educativo; 2) trabajo previo en el aula y evaluación según el sexo; 3) trabajo previo en el aula y evaluación según el tipo de centro escolar; 4) Profesorado que realizan trabajo previo en el aula y evaluación según su grupo de edad y; 5) trabajo previo en el aula y evaluación según el museo visitado.

\section{1) Trabajo previo en el aula y evaluación según el nivel educativo}

En la tabla 3 se puede observar que los profesores del nivel educativo de Bachillerato y Secundaria (100\% y $70.6 \%$ respectivamente) son los que realizan un trabajo previo con sus alumnos antes de realizar una visita al museo. Por su parte, en dicha tabla, vemos que los profesores del nivel educativo de Infantil y Adultos (100\% y 100\% respectivamente) son los que después de la visita más evalúan con sus alumnos lo que éstos han trabajado en el museo. Sin embargo, una mayoría amplia de los profesores de Bachillerato (66.7\%) no lleva cabo ningún tipo de evaluación con sus alumnos después de su visita a un museo.

TABLA 3. Trabajo previo en el aula y evaluación según el nivel educativo

\begin{tabular}{cccccc}
\cline { 3 - 6 } & & \multicolumn{2}{c}{ Trabajo Previo } & \multicolumn{2}{c}{ Evaluación } \\
\cline { 3 - 6 } & & Sí & No & Sí & No \\
\hline \multirow{4}{*}{$\begin{array}{c}\text { Nivel } \\
\text { educativo }\end{array}$} & Primaria & $67.4 \%$ & $32.6 \%$ & $72.7 \%$ & $27.3 \%$ \\
\cline { 2 - 6 } & Secundaria & $70.6 \%$ & $29.4 \%$ & $76.5 \%$ & $23.5 \%$ \\
\cline { 2 - 6 } & Adultos & $50 \%$ & $50 \%$ & $100 \%$ & $0 \%$ \\
\cline { 2 - 6 } & Bachillerato & $100 \%$ & $0 \%$ & $33.3 \%$ & $66.7 \%$ \\
\cline { 2 - 6 } & Infantil & $60 \%$ & $40 \%$ & $100 \%$ & $0 \%$ \\
\hline
\end{tabular}

2) Trabajo previo en el aula y evaluación según el sexo

Con respecto a si es un profesor o una profesora quien realiza un trabajo previo en sus aulas antes de la visita al museo, observamos en la tabla 4 que el $72.5 \%$ de las mujeres frente al $55 \%$ de los hombres realizan un trabajo previo en sus aulas antes de realizar la visita a los museos. También, después de la visita al museo, podemos observar que son las mujeres (78\%, frente al 63.2\% de los hombres) quienes trabajan más la evaluación del museo en sus aulas.

TABLA 4. Trabajo previo en el aula y evaluación según el sexo.

\begin{tabular}{cccccc}
\cline { 3 - 6 } & \multicolumn{2}{c}{ Trabajo Previo } & \multicolumn{2}{c}{ Evaluación } \\
\cline { 2 - 6 } & Sí & No & Sí & No \\
\hline \multirow{2}{*}{ Sexo } & Hombre & $55 \%$ & $45 \%$ & $63.2 \%$ & $36.8 \%$ \\
\cline { 2 - 6 } & Mujer & $72.5 \%$ & $27.5 \%$ & $78 \%$ & $22 \%$ \\
\hline
\end{tabular}


3) Trabajo previo en el aula y evaluación según el tipo de centro escolar

Comparando las respuestas por tipos de centro escolar (tabla 5), los porcentajes de los profesores que hacen trabajo previo tanto de los centros públicos como de los centros concertados o privados son similares (68.7 \% frente al $67.8 \%$ ). En cuanto a la evaluación, tampoco hay grandes diferencias, aunque los profesores de la pública parecen practicarla más (76.5 frente al $74.1 \%$ ).

TABLA 5. Trabajo previo en el aula y evaluación según el tipo de centro escolar

\begin{tabular}{ccccc}
\cline { 2 - 5 } & \multicolumn{2}{c}{ Trabajo Previo } & \multicolumn{2}{c}{ Evaluación } \\
\cline { 2 - 5 } & Sí & No & Sí & No \\
\hline Centro público & $68.7 \%$ & $31.3 \%$ & $76.5 \%$ & $23.5 \%$ \\
\hline Centro concertado o privado & $67.8 \%$ & $32.2 \%$ & $74.1 \%$ & $25.9 \%$ \\
\hline
\end{tabular}

4) Profesorado que realiza trabajo previo en el aula y evaluación según su grupo de edad

En la comparativa de grupos de edad (tabla 6), se cumple la premisa a mayor edad mayor implicación, un 94.7 \% de los más mayores dijeron hacer un trabajo previo, frente al 56 y el 50 \% de los grupos de edad precedentes; tan sólo los que no dijeron la edad se acercan a los mayores, con un $81.8 \%$.

Los resultados que vinculan edad y evaluación están más igualados. De hecho, se puede apreciar (tabla 6) que los que más evalúan son el grupo de edad entre 40 y 54 años con un $79.2 \%$ de respuestas afirmativas, seguidos por los más mayores con un 77.8 \% y por último los más jóvenes con un 61.1\%. Como vemos, hay diferencias entre el trabajo previo y la evaluación, el único grupo que no cambia su respuesta es el que no dijo su edad, que continúa con un $81.8 \%$ de respuestas afirmativas.

TABLA 6. Profesorado que realiza trabajo previo en el aula y evaluación según su grupo de edad.

\begin{tabular}{lcccc}
\hline Rangos de edad & No se sabe & $25-39$ & $40-54$ & $55-65$ \\
\hline$N(\%)$ & $14.7 \%$ & $24 \%$ & $36 \%$ & $25.3 \%$ \\
\hline Trabajo previo & $81.8 \%$ & $50 \%$ & $56 \%$ & $94.7 \%$ \\
\hline Evaluación & $81.8 \%$ & $61.1 \%$ & $79.2 \%$ & $77.8 \%$ \\
\hline
\end{tabular}

\section{5) Trabajo previo en el aula y evaluación según el museo visitado.}

Para analizar el trabajo previo en el aula y evaluación según el museo visitado hemos vuelto a ordenar según el número de visitas los resultados y, exceptuando el caso del Museo Santa Clara con un solo profesor y su grupo de alumnos, los mismos coinciden en que el trabajo previo supera siempre los dos tercios de las respuestas, oscilando entre el 66\% del Helga de Alvear y el 83.3\% del Arqueológico de Badajoz; tan sólo el Vostell, muestra unos índices de trabajo previo por debajo de la media, con un 25 \%. En cuanto a la evaluación, los resultados son más dispares, si bien, los profesores que visitan los centros más centrados en lo contemporáneo suelen evaluar más que los que recorren los centros más centrados en otras etapas de la Historia. La mayoría de los museos visitados muestran índices de evaluación superiores al 60\%, siendo el Helga de Alvear con el 100\% y el MEIAC con el 87.1 \% los más destacados; en el extremo contrario, el Arqueológico de Badajoz sólo muestra un 33.3\% de intención evaluadora de los profesores visitantes hacia su grupo de alumnos. 
TABLA 7. Trabajo previo en el aula y evaluación por parte del profesorado según el museo visitado

\begin{tabular}{cccccccc}
\hline Museos & MEIAC & $\begin{array}{c}\text { González } \\
\text { Santana }\end{array}$ & $\begin{array}{c}\text { Museo } \\
\text { de } \\
\text { Cáceres }\end{array}$ & $\begin{array}{c}\text { Arqueológico } \\
\text { de Badajoz }\end{array}$ & $\begin{array}{c}\text { Helga } \\
\text { de } \\
\text { Alvear }\end{array}$ & Vostell & $\begin{array}{c}\text { Santa } \\
\text { Clara }\end{array}$ \\
\hline $\begin{array}{c}\text { Trabajo } \\
\text { previo }\end{array}$ & $68.7 \%$ & $69.2 \%$ & $72.7 \%$ & $83.3 \%$ & $66 \%$ & $25 \%$ & $\begin{array}{c}100 \\
\%\end{array}$ \\
\hline Evaluación & $87.1 \%$ & $76.9 \%$ & $63.6 \%$ & $33.3 \%$ & $100 \%$ & $75 \%$ & $0 \%$ \\
\hline
\end{tabular}

\section{CONCLUSIONES}

El perfil del profesorado que visita los museos extremeños en horas de clase es el de una mujer de mediana edad, que trabaja en Badajoz, con experiencia en docencia y en visitar museos, que quiere repetir la visita con sus alumnos al año siguiente, que está satisfecha de lo que oferta y proporciona educativamente este tipo de centros culturales, y que ha preparado la visita con antelación y piensa evaluar posteriormente lo aprendido, aunque sea de modo informal.

Por lo tanto, ante la disyuntiva de si la actitud del profesor ha sido pasiva o activa en la visita al museo, habría que decir lo segundo, que el profesor se involucra y da importancia a este tipo de experiencias.

Los resultados muestran que son los profesores más mayores (entre 45 y 65 años) y las mujeres los que mejor preparan su visita al centro, y le dan más importancia a la evaluación. Mientras, los hombres, especialmente los más jóvenes (entre 25 y 45 años), son los más inclinados a la improvisación. No evaluar puede significar no dar importancia a la visita al museo.

A pesar de ello, debemos ser conscientes que los resultados tienen un margen de error importante; primero cuando las respuestas de algunas variables son escasas, y segundo debido a la idiosincrasia del género humano, del que los profesores forman parte. Todo parece indicar que la preparación previa y la evaluación posterior a la visita no son tan intensas como deberían. Las respuestas que los alumnos dan en sus encuestas respectivas, que están siendo objeto de un estudio complementario (en proceso) parecen indicar que la preparación previa y la evaluación posterior a la visita no son tan intensas como deberían..

Aunque la visita guiada es la actividad más repetida en los museos, mostrando los profesores su satisfacción con los guías que la realizan, por su profesionalidad y capacidad para con los menores, muchos docentes manifiestan que les gustaría que les ofertaran talleres o propuestas más activas y participativas.

Se echa en falta que los profesores diseñen sus propias actividades o ejercicios para el alumnado, lo que podría ayudar mucho a los museos y a otros docentes. No creemos que sea sólo responsabilidad del museo esta tarea. En este sentido, no es común la preparación de una unidad didáctica específica, que los profesores podrían redactar. Corroboramos la idea de Arbués y Naval (2014) al considerar que sería bueno fomentar algún encuentro entre profesores y técnicos para poder llevar a cabo trabajos conjuntos que enriquezcan estas actividades. Sligmann (2014) corrobora esta idea de propiciar nexos e intercambio de conocimientos con museos y profesores, para la mejora de la práctica de enseñanza de los museos.

Hay que seguir insistiendo, sobre todo con los alumnos de infantil y primaria, en vincular lo educativo y lo lúdico, labor muy apropiada para la educación no formal y que los profesores no siempre logran fomentar con su conocimiento y dinamismo. La visita a los museos puede complementar, de un modo apropiado, la visión que del patrimonio se ofrece en los libros de texto (Cuenca y López, 2014). 
Como recomendaciones, parece lógico que el museo sea visitado por el profesor previamente de manera individual, para preparar la visita posterior con sus alumnos. En segundo lugar, es importante que los profesores planifiquen la visita didáctica teniendo en cuenta el currículo escolar, y no la piensen sólo como una actividad extraescolar, sino formando parte del proceso de aprendizaje, para así incardinar su contenido con la programación de la materia que imparte. Muchos de los encuestados así lo hacen.

Y por último, como ya se alertaba hace una década, es necesario también incentivar el apoyo o los apoyos que se facilitan al profesor (Ravé, 2006). De hecho, Chong y Smith (2017) consideran que los museos online juegan un papel importante en la tendencia de combinar el aprendizaje formal e informal en el sector de la educación, ya que los medios digitales se han convertido en una fuente esencial de información para muchos y, por lo tanto, sirven de trampolín para el aprendizaje.

Varios de los museos aquí estudiados se toman muy en serio la faceta didáctica que pueden ejercer, y han elaborado materiales muy abundantes que pueden descargarse de sus respectivas páginas webs oficiales (Santibáñez, 2006), lo que ayuda y mucho a los profesores visitantes. No obstante, hay que partir de la idea de que estos museos tienen unos presupuestos limitados, debido a su carácter regional, y que no pueden compararse con la labor que realizan otros museos mayores, estatales, que cuentan muchas veces con la colaboración de otras entidades para la elaboración de actividades y talleres didácticos.

Los profesores no son sólo vigilantes o acompañantes, se muestran interesados y fomentan este tipo de visitas. Los museos les facilitan la labor en muchos casos, organizando visitas guiadas, talleres, cuadernillos didácticos, fichas, etc.

\section{Referencias}

Arbués, E. y Naval, C. (2014). Los museos como espacios sociales de educación. Estudios sobre Educación, 27, 133-151. DOI: 10.15581/004.27.133-151.

Calaf, R., San Fabián, J. L. y Gutiérrez, S. (2017). Evaluación de programas educativos en museos: una nueva perspectiva. Bordón. Revista de pedagogía, 69(1), 45-65.

Caldera de Castro, M. P. (1998). La acción del Museo Nacional de Arte Romano respecto a la enseñanza y la divulgación. Iber. Didáctica de las ciencias sociales, geografía e historia, 15, 57-70.

Caldera de Castro, M. P. y Nodar, R. (2005). Programas públicos y programas educativos de la Red de Museos de Extremadura. RdM. Revista de Museología, 32, 35-41.

Chong, C. y Smith, D. (2017). Interactive Learning Units on Museum Websites. Journal of Museum Education, 1-10. DOI: 10.1080/10598650.2017.1301626

Colom, A. J. (2005). Continuidad y complementariedad entre la educación formal y no formal. Revista de Educación, 338, 9-22.

Cuenca, J. M. y López, I. (2014). La enseñanza del patrimonio en los libros de texto de Ciencias Sociales, Geografía e Historia para ESO. Cultura y Educación, 26(1), 19-37.

Eshach, H. (2006). Science Literacy in Primary Schools and Pre-Schools. Springer: Dordrecht.

Fontal, O. (2016). El Observatorio de Educación Patrimonial en España. Cultura y Educación, 28(1), 254-266. DOI: 10.1080/11356405.2015.1110374.

Kisiel, J. F. (2003). Teachers, museums and worksheets: a closer look at a learning experience. Journal of Science Teacher Education, 14(1), 3-21.

Molina, S. y Muñoz, R. E. (2016). La opinión del profesorado de Educación Secundaria sobre el papel del patrimonio en la enseñanza formal de las ciencias sociales: un estudio de caso. Revista Complutense de Educación, 27(2), 863-880. DOI: 10.5209/rev_RCED.2016.v27.n2.48411. 
Morentin, M. (2015). La formación del profesorado para un uso didáctico de los museos de ciencia. RdM. Revista de Museología, 63, 49-58.

Pol, E. y Asensio, M. (2006). La historia interminable: una visión crítica sobre la gestión de audiencias infantiles en los museos. Mus-A: Revista de los museos de Andalucía, 6, 10-20.

Porta, A. y Mato, M. C. (2016). Preferencias y necesidades del profesorado o acompañante de grupo que visita un Museo Interactivo de Ciencias. Revista Eureka sobre enseñanza y divulgación de las ciencias, 13(3), 511-526. DOI: 10.25267/Rev_Eureka_ensen_divulg_cienc.2016.v13.i3.01.

Ravé, J. L. (2006). Educación en el museo. Mus-A: Revista de los museos de Andalucía, 6, 26-31.

Sánchez, M. C. (2013). La relación museo-escuela: tres décadas de investigación educativa. En C. Aguirre (Ed.), El museo y la escuela. Conversaciones de complemento (pp. 11-22). Medellín (Colombia): Sello Explora-Parque Explora.

Santibáñez, J. (2006). Los museos virtuales como recurso de enseñanza-aprendizaje. Comunicar: Revista científica iberoamericana de comunicación y educación, 27, 155-162.

Sligmann, T. (2014). Learning Museum. A Meeting Place for Pre-Service Teachers and Museums. Journal of Museum Education, 39(1), 42-53. DOI: 10.1080/10598650.2014.11510794.

Verde, A. M. (1993). El uso de los cuestionarios en el museo: encuestas ¿para qué? Boletín de la ANABAD, 43(3-4), 257-262.

VV.AA. (2008). El potencial educativo de los museos, análisis de la oferta didáctica de los museos para la educación primaria y secundaria. Copenhague: Dirección General de Patrimonio Cultural de Dinamarca.

\section{Cómo CITAR ESTE ARTíCULO}

González González, J.M. y Becerra Traver, M.T. (2019). Educación no formal. El papel del profesorado en las visitas didácticas a los museos. Didáctica de las ciencias experimentales y sociales, 37, 37-50. DOI: 10.7203/DCES.37.13307 


\section{Anexo 1. Cuestionario para el profesorado}

Este cuestionario está destinado a recoger información sobre la experiencia docente en relación con los museos. Se trata de un estudio llevado a cabo por investigadores de la Universidad de Extremadura. Su labor como maestro/profesor es importante. Garantizamos la confidencialidad de los datos recogidos y agradecemos el interés y el tiempo disponible para responder a este cuestionario.

Indique el nombre del museo que ha visitado:

Fecha:

\section{Datos de identificación}

Colegio o Instituto:

Especialidad:

Edad: Sexo: Hombre Mujer

\section{Visita al museo:}

Es mi primera visita a este museo: sí $\square$ No $\square$ Es mi primera visita a un museo con el colegio o instituto: Tengo pensado repetir la visita el curso que viene: Sí Sí He trabajado previamente con los alumnos algo en clase antes de venir al museo: En caso afirmativo, explique brevemente:

Sí

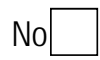

Piensa evaluar en clase lo aprendido hoy en el museo: $\quad$ Sí $\quad$ No $\square$ En caso afirmativo, indique cómo:

Rodee con un círculo qué han hecho sus alumnos hoy en el museo (pueden señalarse varias opciones):
- visita guiada
- juegos
- ordenador
- taller
- audiovisual
- redacción

- hoja de ejercicios

- juego de roles 0 dramatización

Si marca la opción otros, especifique:

3. Información sobre la visita a este museo. Marque con una cruz en la casilla correspondiente, siendo 1=Totalmente en desacuerdo, 2=En desacuerdo, 3=Ni de acuerdo ni en desacuerdo, 4=De acuerdo y 5=Totalmente de acuerdo.

\begin{tabular}{|l|c|c|c|c|c|}
\hline Guía & $\mathbf{1}$ & $\mathbf{2}$ & \multicolumn{1}{|c|}{$\mathbf{3}$} & $\mathbf{4}$ & $\mathbf{5}$ \\
\hline $\begin{array}{l}\text { La información recibida por el guía ha sido correcta y adaptada a mis } \\
\text { alumnos. }\end{array}$ & & & & \\
\hline La organización de la visita me ha gustado. & & & \\
\hline Recursos y contenido & & & & & \\
\hline La duración de la visita es la adecuada. & & & & \\
\hline Los textos y objetos se adaptan a mis alumnos. & & & & & \\
\hline Se han hecho actividades didácticas. & & & & & \\
\hline Valoración global & & & & \\
\hline Como profesor/a, me ha gustado lo que se ha propuesto por el museo. & & & & \\
\hline $\begin{array}{l}\text { He aprendido cuestiones que desconocía enriqueciéndome como } \\
\text { profesor. }\end{array}$ & & & & & \\
\hline Recomendaré la visita a compañeros/familiares/amigos. & & & \\
\hline
\end{tabular}

La actividad que más me ha gustado ha sido:

La actividad que me gustaría que mis alumnos hicieran cuando visitaran un museo es: 
